\title{
Evaluación de las características físicas y sensoriales de licor de cacao asociadas a modelos de siembra
}

\author{
Edith Moreno-Martínez ${ }^{1}$ \\ Óscar Mauricio Gavanzo-Cárdenas ${ }^{2}$ \\ Fabian Alberto Rangel-Silva ${ }^{3}$
}

Fecha de recepción: 23 de junio de 2019

Fecha de aceptación: 31 de julio de 2019

\section{Resumen}

El fomento de cultivos comerciales de cacao ha conllevado el aumento del uso de modelos de siembra, ha favorecido la organización genética y la estandarización del proceso de fermentación, y ha optimizado la calidad, según las necesidades del mercado y del consumidor. Teniendo en cuenta esto, se realizó la evaluación física y sensorial de cuatro modelos de siembra en dos municipios: Arauquita, del departamento de Arauca, y San Vicente de Chucurí, del departamento de Santander. Los modelos evaluados fueron los siguientes: Modelo 1 (M1), autocompatibles: FSV41, FTA2, FEAR5; Modelo 2 (M2), tamaño de grano: FSA12, FSA13, FLE3, FLE2; Modelo 3 (M3) tolerancia monilia: CCN51, FEC2, CAU39; Modelo 4 (M4) monoclonal: CCN51. La evaluación física y sensorial se llevó a cabo por el panel de catación, donde se analizaron: humedad (\%), índice de grano (I.G), fermentación (\%); y para el licor de cacao se describieron las características sensoriales de sabores básicos: cacao, acidez, amargor, astringencia; específicos: fruta fresca, fruta seca, floral, nuez, dulce y otros. Se hizo la prueba no paramétrica de Wilcoxon, para determinar si existían diferencias significativas entre cada una de las variables de los modelos evaluados. Adicionalmente se hizo un análisis de componentes principales (ACP) para evaluar la relación entre las variables evaluadas y las características de los modelos. Según los resultados, el índice de grano más alto fue dado al M1. Sensorialmente, el M1 se caracterizó por tener altos valores en sabor cacao, fruta fresca, fruta seca, nuez y dulce. El M4 se destacó por altas intensidades de acidez, amargor y sensación de astringencia. La calidad física y sensorial de los modelos de siembra en las dos localidades no presentó diferencias significativas.

Palabras clave: análisis estadístico; cacao; evaluación comparativa; fermentación; selección de las plantas.

\footnotetext{
1 Federación Nacional de Cacaoteros - Fedecacao (San Vicente de Chucurí-Santander, Colombia). investigacion@fedecacao.com.co. ORCID: 0000-0003-2626-7443.

${ }^{2}$ Federación Nacional de Cacaoteros - Fedecacao (San Vicente de Chucurí-Santander, Colombia). ORCID: 0000-0001-5847-1284.

${ }^{3}$ Asesor estadístico independiente (San Vicente de Chucurí-Santander, Colombia). ORCID: 0000-0002-7429$\underline{4748}$.
} 


\section{Evaluation of the Physical and Sensory Characteristics of Cocoa Liquor Associated with Sowing Models}

\section{Abstract}

The promotion of commercial crops of cocoa has increased the use of sowing models, favoring the genetic organization, standardization of the fermentation process and optimizing the quality, obeying the needs of the market and consumer. Taking this into account, the physical and sensory evaluation of four sowing models was carried out in two municipalities: Arauquita (Arauca) and San Vicente de Chucurí (Santander). The models evaluated were the following: Model 1 (M1), self-compatible: FSV41, FTA2, FEAR5; Model 2 (M2), grain size: FSA12, FSA13, FLE3, FLE2; Model 3 (M3), monilia tolerance: CCN51, FEC2, CAU39; Monoclonal Model 4 (M4): CCN51. The physical and sensorial evaluation was carried out by the tasting panel, where also were analyzed: humidity (\%), grain index (IG), fermentation (\%), and for the cocoa liquor the description of the sensory characteristics of basic flavors was made: cocoa, acidity, bitterness, astringency; specific: fresh fruit, dried fruit, floral, walnut, sweet and others. The non-parametric Wilcoxon test was performed to determine if there were significant differences between each of the variables of the evaluated models. Additionally, a Principal Components Analysis (PCA) was performed to assess the relationship between the evaluated variables and the characteristics of the models. According to the results, the highest grain index was given to M1. In a sensory meaning, the M1 was characterized by having high values in cocoa flavor, fresh fruit, dried fruit, walnut and sweet. The M4 was highlighted by high intensities of acidity, bitterness and astringency sensation. The physical and sensory quality of the sowing models in the two localities did not present significant differences.

Keywords: cacao; comparative evaluation; fermentation; plant breeding; statistical analysis.

\section{Para citar este artículo:}

Moreno-Martínez, E., Gavanzo-Cárdenas, O. M., \& Rangel-Silva, F. A. (2019) Evaluación de las características físicas y sensoriales de licor de cacao asociadas a modelos de siembra. Ciencia y Agricultura, 16(3), $75-90$ https://doi.org/10.19053/01228420.v16.n3.2019.9890.

Esta obra está bajo licencia internacional Creative Commons Reconocimiento 4.0

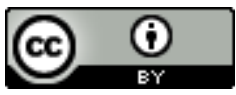




\section{INTRODUCCIÓN}

El cultivo de cacao en Colombia ha sido catalogado como fino y de aroma reconocido por la Organización Internacional del Cacao (International Cocoa Organization [ICCO], 2016), característica dada por la diversidad de los materiales cultivados y por ser el centro de origen de la especie (Motamayor et al., 2008).

Buscando dar mayor eficiencia al recurso genético existente en Colombia, la Compañía Nacional de Chocolates y la unión temporal Cacao de Colombia Uno (Aranzazu et al., 2009b), iniciaron estudios de compatibilidad sexual, cuyos resultados indican que Colombia cuenta con una matriz consolidada por 21 materiales comerciales, entre introducidos y regionales, la cual ha servido para la propuesta de modelos de siembra.

Utilizando la matriz de compatibilidad sexual y de acuerdo con características especiales como tamaño de grano, tolerancia a monilia y escoba, alta producción y materiales autocompatibles, Fedecacao-Corpoica (Aranzazu et al., 2009a) determinaron que con cultivos comerciales bajo modelos de siembra se mejora la capacidad productiva, se facilita el manejo de enfermedades, se disminuyen costos en poda, se renueva fácilmente copa por cambio de material y se benefician materiales por exigencias de calidad en forma separada.

La calidad de los granos de cacao se debe principalmente a su composición aromática, siendo el genotipo el factor de mayor importancia; sin embargo, las condiciones agroclimáticas en las que se desarrolla el cultivo, los procesos de cosecha, fermentación, secado y procesamiento, son factores relevantes en la formación de componentes volátiles y no volátiles que definen la calidad del producto final (Afoakwa, Paterson, Fowler \& Ryan, 2008; Brito, 2007; Sim et al., 2015).

No obstante, uno de los procesos que ayudan a determinar la calidad final del cacao es la fermentación, la cual se da en dos etapas: anaeróbica y aeróbica. Después de 48 a 96 horas, los diferentes microorganismos crecen y degradan el etanol en ácido acético, que entra en los cotiledones favorecidos por acción de la temperatura. Según Afoakwa, Kongor, Takrama y Budu (2013), las consecuencias son la disminución del pH del grano, la muerte del germen y, la más importante, el inicio de reacciones bioquímicas que dan origen a los precursores del aroma y sabor (Contreras, Ortiz, Graziani \& Parra, 2004). Hay que anotar que han sido identificados en los granos de cacao alrededor de 600 compuestos de sabor (Crafack et al., 2014).

De acuerdo con las características genéticas del cacao y los procesos de beneficio, el producto final de cacao puede reflejar características organolépticas favorables. En el cacao se han identificado sabores básicos, como dulce, amargo, ácido y la sensación astringente (Fisher \& Scott, 2000). Otros aromas identificados en el cacao son los denominados específicos, como cacao, floral, frutal, nuez, entre otros (Sánchez, 2007). 
En este contexto, el objetivo de esta investigación consistió en la evaluación y comparación de las características físicas y sensoriales, asociadas a modelos de siembra de cacao en dos municipios, Arauquita y San Vicente de Chucurí.

\title{
II. MATERIALES Y MÉTODOS
}

\begin{abstract}
A. Ubicación
La investigación se desarrolló en dos granjas de propiedad de Fedecacao: Granja Villa Mónica, ubicada en el municipio de San Vicente de Chucuri, Santander, Colombia (N 6 $51^{\prime} 48.265^{\prime \prime}$, W $\left.73^{\circ} 24^{\prime} 47.971^{\prime \prime}\right)$ localizada a $879 \mathrm{msnm}$, con una temperatura promedio de $23{ }^{\circ} \mathrm{C}$ y una humedad relativa promedio de $81 \%$; y la Granja Santa Elena, ubicada en el municipio de Arauquita, Arauca, Colombia (N $07^{\circ} 01^{\prime} 35,1^{\prime \prime}, \mathrm{W} 071^{\circ} 23^{\prime} 31,6^{\prime \prime}$ ), localizada a $162 \mathrm{msnm}$, con una temperatura promedio de $28{ }^{\circ} \mathrm{C}$ y una humedad relativa promedio de $75 \%$.
\end{abstract}

\section{B. Diseño experimental}

Se efectuaron cuatro tratamientos con tres repeticiones, compuestos por los siguientes genotipos: Modelo 1 (M1) autocompatibles: FSV41, FTA2, FEAR5; Modelo 2 (M2) tamaño de grano: FSA12, FSA13, FLE3, FLE2; Modelo 3 (M3) tolerancia monilia: CCN51, FEC2, CAU39; Modelo 4 (M4) monoclonal: CCN51.

\section{Manejo cosecha y poscosecha}

Se cosecharon mazorcas maduras y sanas evitando herir los granos. No se incluyeron: placenta, granos afectados por enfermedades y residuos de cáscara. La masa fermentante se dispuso en un cajón de madera $(3 \mathrm{~m}$ de largo, $0.90 \mathrm{~m}$ de ancho y $0.80 \mathrm{~m}$ de alto) en un lugar cerrado para evitar las corrientes directas de aire. Las muestras se fermentaron en el centro de una masa de 500 kilos de cacao, usando la técnica de microfermentación, depositando los granos de cacao húmedos en mallas de nylon (Eskes et al., 2012). Las muestras se dispusieron de forma que se evitó que los granos se salieran al momento de la remoción de la masa (Fig. 1). Transcurridas 48 horas desde el inicio de la fermentación, se realizó la primera remoción de la masa, cambiando las bolsas de posición (Fig. 1). 

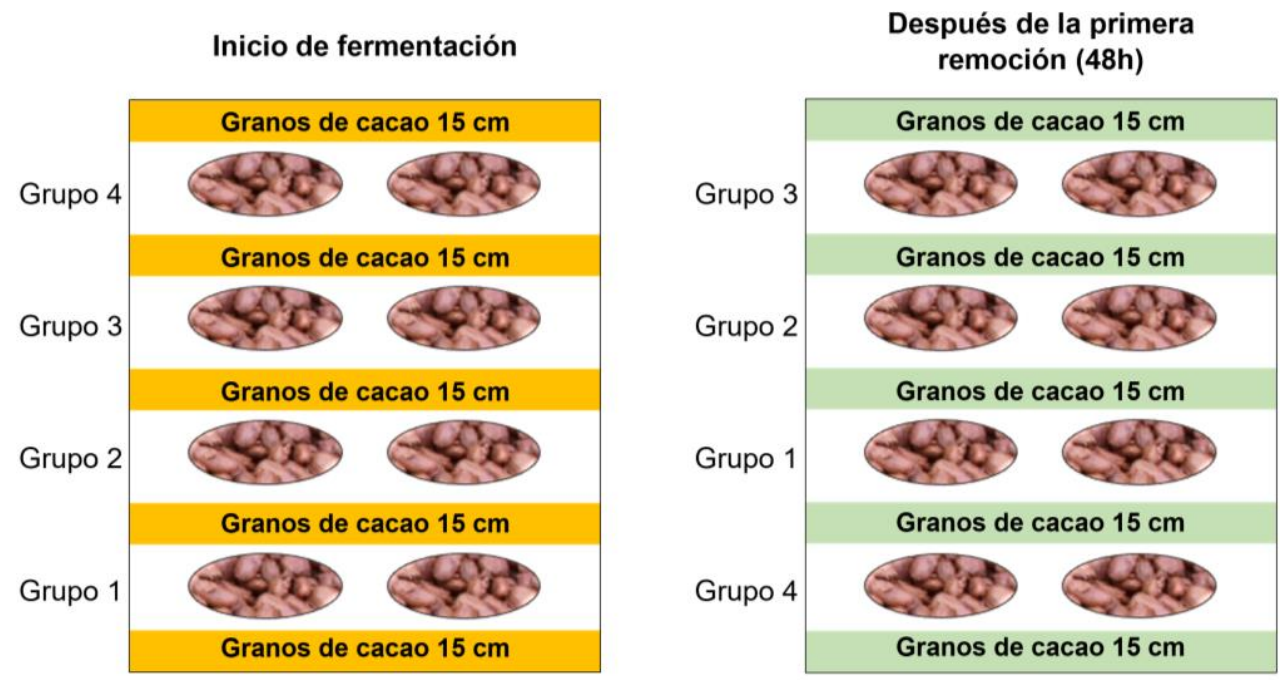

Fig. 1. Disposición en el cajón de las muestras de cacao para microfermentación.

A las 72, 96 y 120 horas del inicio de la fermentación, el proceso se replicó; cumplidas 144 horas (6 días), la masa fue retirada del cajón fermentador para dar inicio a la etapa de secado.

El secado fue realizado en casa elba, plataforma de madera con techo corredizo, colocando la masa de las muestras de cacao separadas en capas de 5 a $7 \mathrm{~cm}$ de espesor. La exposición solar varió entre 3 a 4 horas/día, los granos fueron removidos para cambiar de posición y uniformizar el secado. De la misma manera se llevó a cabo el proceso durante 7 días, disminuyendo el espesor de la masa y ampliando con los días la exposición solar, realizando entre dos a cuatro remociones por día para homogeneizar el grado de secado. La finalización del secado de la masa es determinada por el porcentaje de humedad.

\section{D. Índice de grano}

Se tomaron tres muestras aleatorizadas de 100 granos, cada una de cada modelo. Cada muestra se pesó y se promedió; este promedio se dividió en 100. El valor obtenido corresponde al índice de grano de cada modelo (Instituto Colombiano de Normalización y Certificación [Icontec], 2012).

\section{E. Humedad (\%)}

El método consistió en determinar el contenido de agua presente en los granos de cacao que han sido fermentados y secados, usando un equipo medidor de humedad marca Agratronix. Se tomaron tres submuestras de cada modelo, donde se calculó la humedad y se promedió. La humedad se redujo mediante exposición directa al sol hasta alcanzar entre $7-7.5 \%$ de humedad, de acuerdo con NTC 1252 (Icontec, 2012).

\section{F. Fermentación (\%)}

Para determinar el porcentaje de fermentación, los granos se clasificaron por categorías: granos de color marrón o café, granos de color marrón y violeta, 
granos totalmente violetas y granos pizarrosas (Stevenson, Corven \& Villanueva, 1993; De Zaan, 2009).

Se dispusieron 100 granos de cacao al azar por cada modelo. Utilizando una guillotina magra 12, se hizo un corte longitudinal a los granos. Posteriormente se inspeccionaron visualmente los granos, contando los granos bien fermentados o marrones, medianamente fermentados o marrones con tonos violeta, granos violetas y pizarrosos. El porcentaje de fermentación se obtuvo de sumar la cantidad de granos bien fermentados con los medianamente fermentados y dividir por 100, que es el número total de granos (De Zaan, 2009; Jiménez et al., 2011, Quintana, Hernández, Rivera \& Moreno, 2018).

\section{G. Evaluación sensorial}

El licor de cacao se preparó sometiendo los granos a los procesos de tostión, descascarillado y molienda hasta obtener el licor.

La evaluación sensorial la realizó el panel de catación de Fedecacao, utilizando la metodología perfil del sabor según la NTC 3929 de 2009 (Icontec, 2009), donde se calificaron los atributos básicos (cacao, acidez, amargor y sensación de astringencia) y los atributos específicos (fruta fresca, fruta seca, floral, nuez, dulce).

\section{H. Análisis estadístico}

Los datos se analizaron estadísticamente mediante la prueba no paramétrica de Wilcoxon con un nivel de significancia del $5 \%$, para evaluar si existían diferencias significativas en cada una de las características sensoriales y físicas entre los cuatro modelos y entre los dos municipios evaluados. Posteriormente se realizó un análisis de componentes principales (ACP), con el fin de describir los atributos sensoriales de cada uno de los modelos, así como los atributos físicos. Los análisis y los gráficos se obtuvieron mediante la interfaz RWizard Software (Guisande, Heine, González-DaCosta \& García-Roselló, 2014).

\section{RESULTADOS Y DISCUSIÓN}

\section{A. Prueba de Wilcoxon para características sensoriales y físicas}
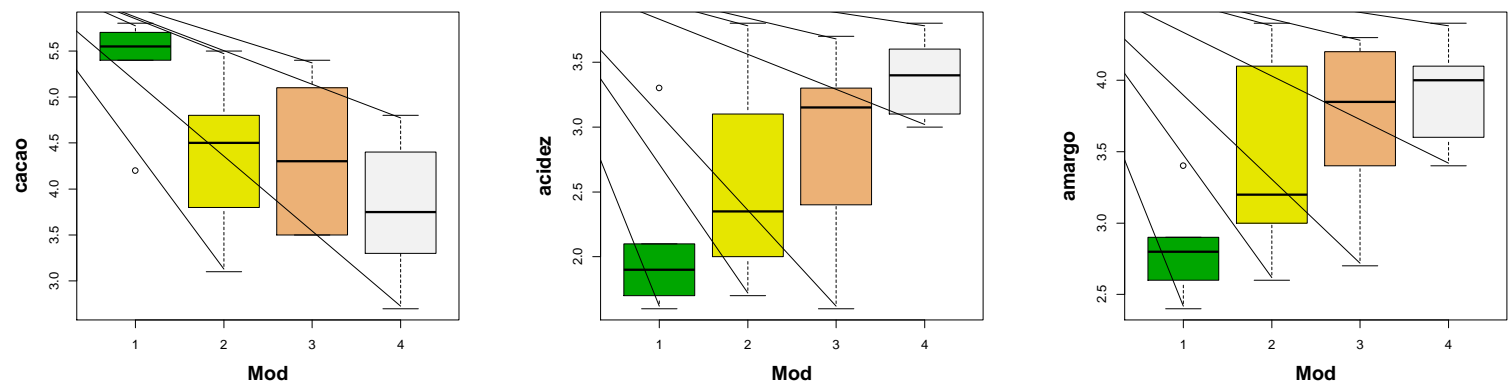

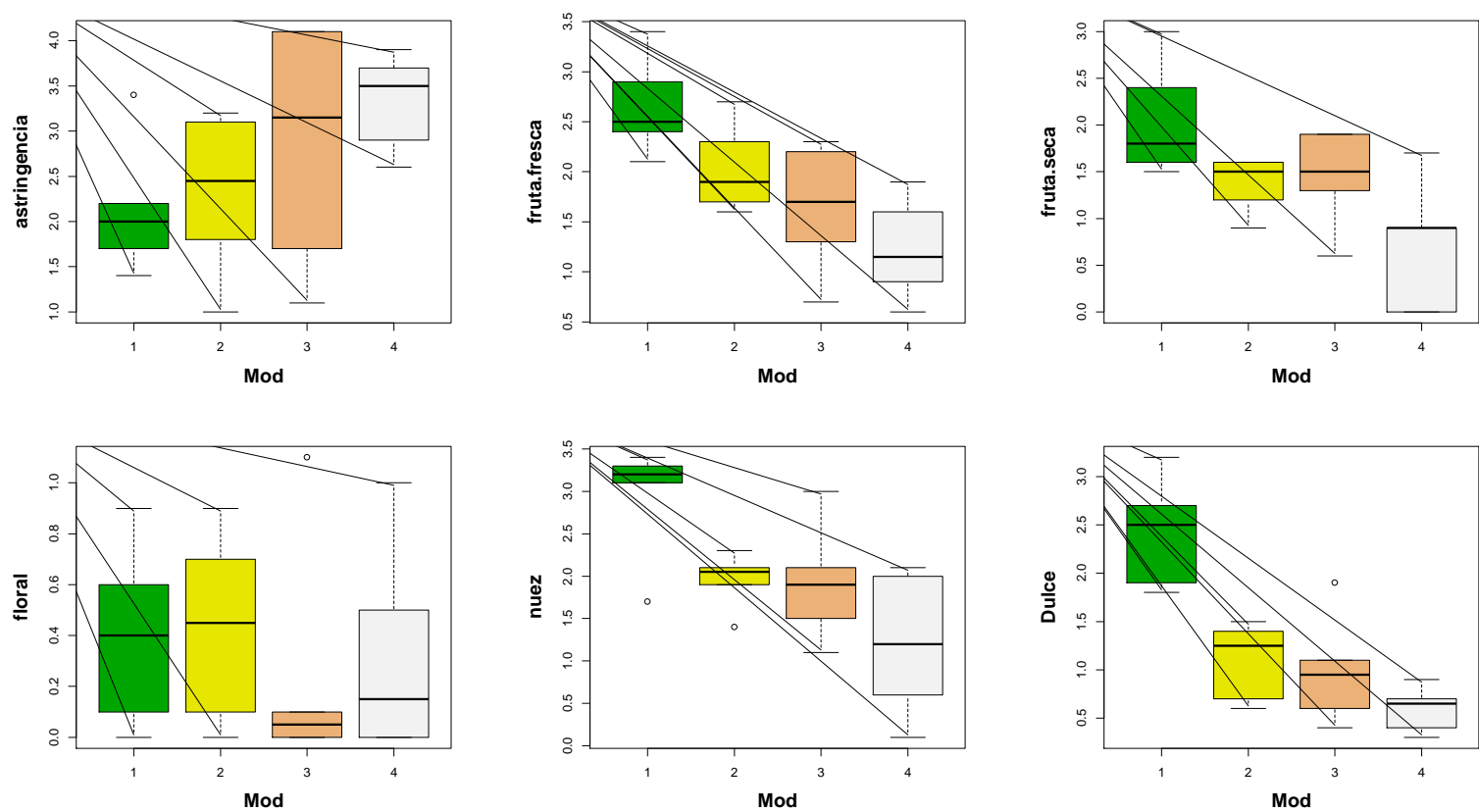

Fig. 2. Gráfico de caja para los modelos y cada atributo sensorial evaluado.

En la Figura 2 se presentan los gráficos de caja de los cuatro modelos y los atributos sensoriales evaluados. El M1 se caracterizó por tener altos valores en sabor a cacao, fruta fresca, fruta seca, nuez y dulce, atributos que comparte cada material que conforma el modelo (Martínez, Moreno, Duarte \& Gavanzo, 2016). Así mismo, se destacó por bajas intensidades de acidez, amargor y sensación de astringencia. El M4, en cambio, presentó características opuestas, es decir, altas intensidades de acidez, amargor y sensación de astringencia y bajos valores de sabor a cacao, fruta fresca, fruta seca, nuez y dulce. En cuanto al sabor floral, no se evidenció una fuerte diferencia entre los modelos evaluados.
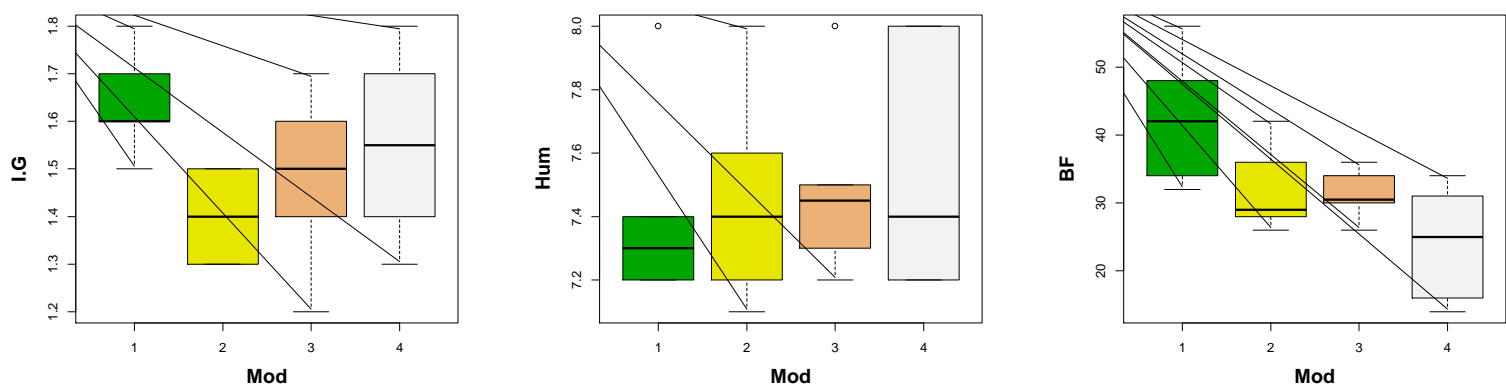

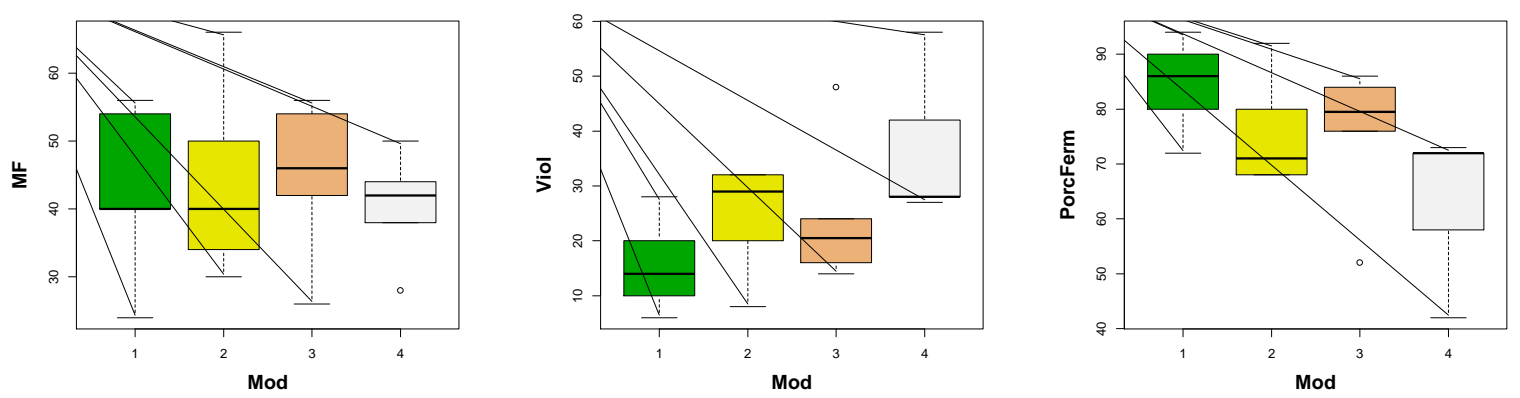

Fig. 3. Gráfico de caja para los modelos y cada atributo físico evaluado.

En cuanto a las características físicas (Fig. 3), el M1 tuvo el mayor índice de grano (IG), un mayor número de granos bien fermentados (BF) y, por ende, un mayor porcentaje de fermentación (PorcFerm). En contraste, el M4 se caracterizó por tener un alto contenido de granos violetas (Viol) y un bajo porcentaje de fermentación (PorcFerm).

En la Tabla 1 se exponen los resultados obtenidos por la prueba de Wilcoxon con un nivel de significancia del $5 \%$ para las variables sensoriales seleccionadas. Dentro de los resultados más relevantes se encontró que el M1 se diferenció del M4 en casi todas las características, exceptuando la característica floral, que fue similar en ambos modelos. El M1 solo presentó diferencias significativas con el M2, en las características de nuez y dulce. El M1 y el M3 mostraron diferencias significativas en las características de cacao, amargo, fruta fresca, nuez y dulce. En cuanto a las características sensoriales del M3, estas no revelaron diferencias significativas con las del M2, ni con las del M4. Finalmente, el M2 se diferenció del M4 por las características de astringencia y fruta seca. Según trabajos realizados en evaluación sensorial (Boza et al., 2014; Menezes et al., 2016), CCN 51 exhibe una cantidad moderada de sabor a cacao y altos niveles de amargor y astringencia, consistentes con el perfil encontrado en las dos localidades.

Tabla 1. Prueba de Wilcoxon para las variables sensoriales y físicas seleccionadas.

\begin{tabular}{|c|c|c|c|c|c|c|c|c|c|}
\hline \multirow{14}{*}{$\begin{array}{l}\frac{0}{3} \\
\frac{3}{1} \\
\varepsilon\end{array}$} & \multirow{10}{*}{ 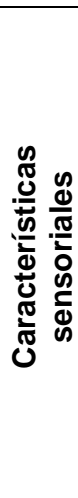 } & & M1: M2 & M3: M1 & M4: M1 & M3: M2 & M4: M2 & M4: M3 & SVC: ARA \\
\hline & & Cacao & 0.05424 & $0.02447^{*}$ & $0.00866^{*}$ & 1 & 0.2963 & 0.229 & 0.4879 \\
\hline & & Acidez & 0.2273 & 0.1712 & $0.01594^{*}$ & 0.5211 & 0.09155 & 0.2963 & 0.8849 \\
\hline & & Astringencia & 0.5887 & 0.4217 & $0.01612^{*}$ & 0.3776 & $0.02597^{\star}$ & 0.8099 & 0.644 \\
\hline & & Amargo & 0.1081 & $0.02977^{*}$ & $0.00621^{*}$ & 0.4848 & 0.1962 & 0.6874 & 0.7067 \\
\hline & & Fruta fresca & 0.0534 & $0.01291^{*}$ & $0.00499^{*}$ & 0.335 & $0.02447^{\star}$ & 0.1797 & 0.8397 \\
\hline & & Fruta seca & 0.0562 & 0.1249 & $0.01895^{\star}$ & 0.6825 & 0.09997 & 0.08927 & 0.4481 \\
\hline & & Floral & 0.9356 & 0.2495 & 0.5136 & 0.2504 & 0.4624 & 0.864 & 0.1567 \\
\hline & & Nuez & $0.0442^{*}$ & $0.02397^{*}$ & $0.01275^{*}$ & 0.8068 & 0.07557 & 0.146 & 0.5231 \\
\hline & & Dulce & $0.00217^{*}$ & $0.01027^{\star}$ & $0.00499^{*}$ & 0.4225 & 0.05256 & 0.1467 & 0.9963 \\
\hline & $\stackrel{0}{=} 0$ & IG & $0.00716^{*}$ & 0.1181 & 0.4608 & 0.285 & 0.1644 & 0.6267 & 0.06887 \\
\hline & 乐 & Hum & 0.8707 & 0.3691 & 0.6792 & 0.8712 & 0.8068 & 1 & 0.9063 \\
\hline & $\bar{g}$ & BF & $0.0449^{*}$ & $0.0245^{*}$ & $0.01027^{*}$ & 0.8079 & 0.2265 & 0.1712 & 0.1734 \\
\hline & & MF & 0.9338 & 0.4657 & 1 & 0.5196 & 1 & 0.4142 & 0.337 \\
\hline
\end{tabular}




\begin{tabular}{|l|c|c|c|c|c|c|c|c|c|}
\hline \multirow{2}{*}{} & Viol & 0.09098 & 0.1994 & $0.01448^{*}$ & 0.4704 & 0.6242 & $0.04383^{*}$ & $0.0453^{*}$ \\
\cline { 3 - 9 } & PorcFerm & 0.09098 & 0.1994 & $0.01448^{*}$ & 0.4704 & 0.6242 & $0.04383^{*}$ & $0.0453^{*}$ \\
\hline
\end{tabular}

$M=$ Modelo; * Diferencias significativas $(p>0.05)$

En cuanto a las características físicas, el M1 se diferenció de los demás modelos por la cantidad de granos bien fermentados (BF). El M2 presentó diferencias significativas con el M1 en cuanto al índice de grano (IG). Por otra parte, tanto la cantidad de granos violetas (Viol) como el porcentaje de fermentación (PorcFerm) fueron características diferenciadoras entre el M1 y el M4. El M2 no se diferenció del M3 ni del M4 en ninguna característica física. Finalmente, el M4 evidenció disimilitud con el M3 por características como el porcentaje de fermentación (PorcFerm) y el número de granos violetas (Viol).

En cuanto a las localidades, la prueba de Wilcoxon no demostró diferencias significativas entre las características sensoriales evaluadas, siendo entonces las características genéticas las determinantes en el perfil sensorial de los licores de cacao obtenidos. Enríquez (1985) indica que la calidad del chocolate depende del genotipo de cacao, de factores medioambientales y de un adecuado proceso de fermentación, aspectos que influyen en la calidad del producto final. Sin embargo, en cuanto a las características físicas, las muestras de San Vicente presentaron mayor cantidad de granos violetas (Viol) y menores porcentajes de fermentación (PorcFerm).

\section{B. Análisis de componentes principales para características sensoriales}

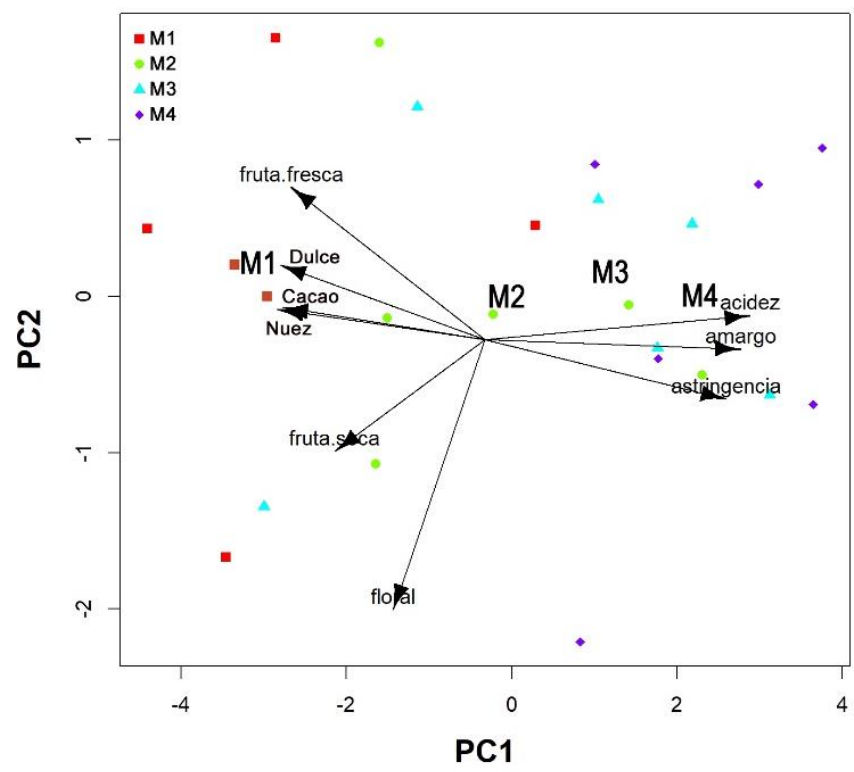

Fig. 4. Análisis de componentes principales (ACP), componentes 1 y 2 , para características sensoriales utilizando como variable categórica el modelo.

Las dos primeras componentes lograron explicar casi el $81 \%$ de la varianza total. En la Tabla 2 se presenta el peso de cada una de las variables en las diferentes componentes. La primera componente (PC1) contrasta las características más 
agradables (cacao, fruta fresca, fruta seca, nuez y dulce), con las poco agradables (acidez, amargor y astringencia). La componente dos (PC2) ayuda a diferenciar los modelos por una característica singular del cacao, como lo es el sabor floral.

Tabla 2. Peso de las variables sensoriales en cada una de las componentes.

\begin{tabular}{|c|c|c|c|c|c|c|c|c|c|}
\hline & PC1 & PC2 & PC3 & PC4 & PC5 & PC6 & PC7 & PC8 & PC9 \\
\hline Cacao & -0.36 & -0.03 & 0.15 & -0.14 & 0.65 & -0.47 & -0.34 & 0.11 & -0.25 \\
\hline Acidez & 0.37 & -0.05 & 0.30 & 0.17 & 0.05 & 0.27 & -0.02 & 0.63 & -0.51 \\
\hline Astringencia & 0.33 & -0.27 & 0.34 & 0.58 & 0.01 & -0.23 & -0.18 & -0.53 & -0.11 \\
\hline Amargo & 0.36 & -0.14 & 0.37 & -0.21 & 0.43 & 0.14 & 0.06 & 0.10 & 0.67 \\
\hline Fruta fresca & -0.34 & 0.28 & 0.19 & 0.17 & 0.11 & 0.68 & -0.47 & -0.20 & 0.04 \\
\hline Fruta seca & -0.28 & -0.40 & 0.58 & -0.45 & -0.46 & 0.00 & -0.05 & -0.07 & -0.09 \\
\hline Floral & -0.18 & -0.81 & -0.41 & 0.16 & 0.12 & 0.25 & -0.11 & 0.13 & 0.07 \\
\hline Nuez & -0.37 & -0.03 & 0.23 & 0.21 & 0.29 & 0.17 & 0.78 & -0.14 & -0.14 \\
\hline Dulce & -0.36 & 0.08 & 0.19 & 0.53 & -0.26 & -0.27 & -0.05 & 0.47 & 0.43 \\
\hline
\end{tabular}

$P C=$ Componente principal

La primera componente estableció que el M1 se identificó por tener mejores atributos sensoriales, asociados a altos valores en características como sabor a cacao, fruta fresca, fruta seca, nuez y dulce. Por el contrario, el M4 se caracterizó por sus sabores intensos de acidez, amargor y sensación de astringencia, del mismo modo como lo hace el M3. Para el caso del M2 se puede decir que posee características promedio de los cuatro modelos evaluados. En cuanto a la segunda componente, los cuatro modelos evaluados son similares en la característica floral, pues se encuentran cercanos al valor promedio para esta variable (Fig. 4).

Con la primera componente, no se encontraron diferencias entre las características de cacao, frutos frescos, frutos secos, nuez dulce, acidez, amargor y sensación de astringencia. Sin embargo, con la segunda componente se logró evidenciar que la característica de sabor floral se encuentra ligeramente marcada en Arauquita (ARAUQ) (Fig. 5). 


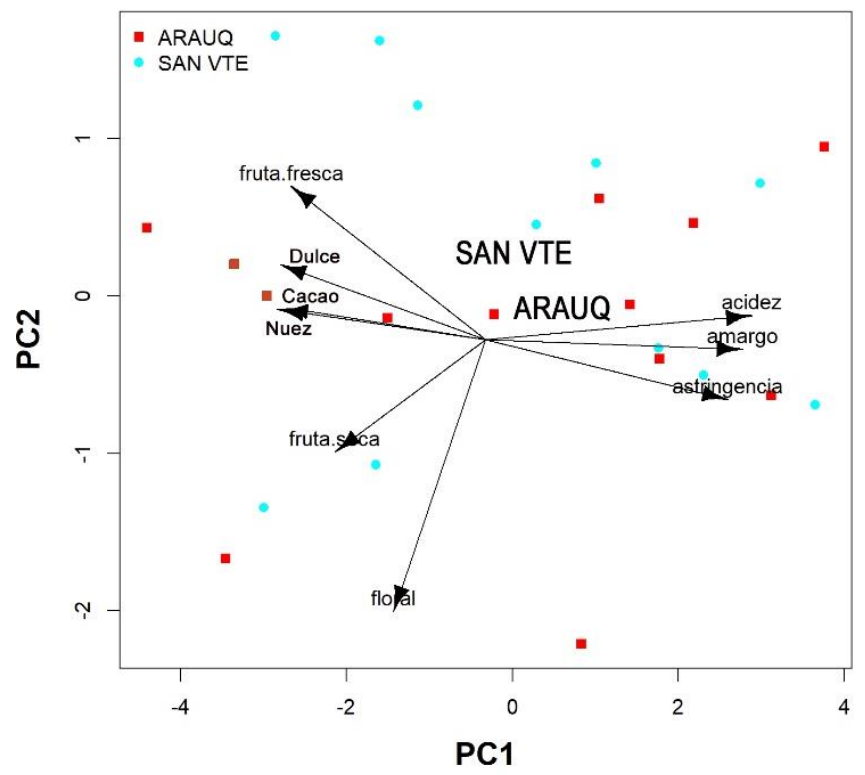

Fig. 5. Análisis de componentes principales -ACP-, componentes 1 y 2, para características sensoriales utilizando la localidad como variable categórica.

\section{Análisis de componentes principales para características físicas}

Las características físicas estudiadas fueron: porcentaje de humedad (Hum), índice de grano (IG) y porcentaje de fermentación (PorcFerm), donde se midieron granos bien fermentados (BF), granos medianamente fermentados (MF), granos violetas (Viol) y granos pizarrosos. Como no se obtuvieron granos pizarrosos, no se incluyeron en el análisis. Con las dos primeras componentes se explica un 76 $\%$ de la varianza total.

De acuerdo con el peso de las variables en cada componente (Tabla 3), la primera componente diferencia los modelos con mayor porcentaje de fermentación y granos medianamente fermentados, contra granos violetas; es decir, que los modelos con altos porcentajes de fermentación poseen mejores características sensoriales en comparación con los modelos que tienen menor porcentaje de fermentación y alta presencia de granos violetas. Para el componente dos se logran distinguir los modelos con mejor índice de grano y granos bien fermentados.

Tabla 3. Peso de las variables físicas en cada una de las componentes.

\begin{tabular}{|c|c|c|c|c|c|c|}
\hline & PC1 & PC2 & PC3 & PC4 & PC5 & PC6 \\
\hline Índice de grano & 0.23 & -0.51 & 0.49 & 0.67 & 0.00 & 0.00 \\
\hline Humedad & 0.30 & 0.03 & -0.81 & 0.51 & 0.00 & 0.00 \\
\hline Bien fermentados & -0.27 & -0.69 & -0.27 & -0.24 & 0.18 & -0.54 \\
\hline Medianamente fermentados & -0.42 & 0.48 & 0.13 & 0.42 & 0.20 & -0.60 \\
\hline Violetas & 0.55 & 0.12 & 0.10 & -0.17 & 0.79 & -0.13 \\
\hline Fermentación (\%) & -0.55 & -0.12 & -0.10 & 0.17 & 0.55 & 0.58 \\
\hline
\end{tabular}

$P C=$ Componente principal 
Con respecto a la primera componente, el M1 presentó mejores características físicas evidenciando altos valores en el número de granos bien fermentados (BF); en cambio, el M4 no mostró las mejores características físicas, pues tuvo una alta cantidad de granos violetas (Viol). En cuanto al segundo componente, el M1 tuvo mayor índice de grano (IG) y mayor cantidad de granos bien fermentados (BF) (Fig. 6).

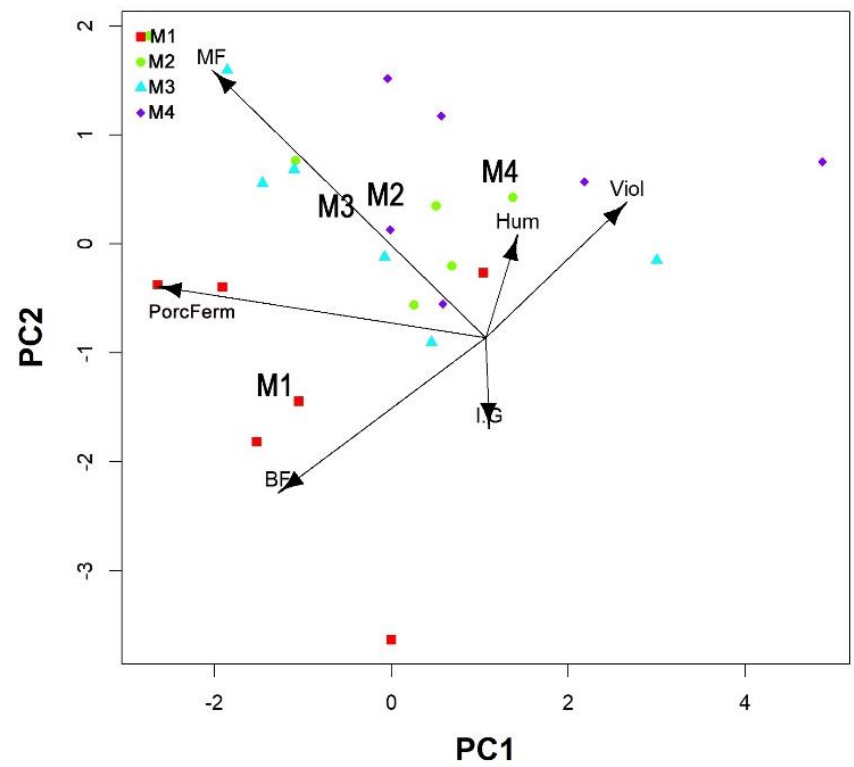

Fig. 6. Análisis de componentes principales (ACP), componentes 1 y 2, para características físicas utilizando como variable categórica el modelo.

Al utilizar la localidad como variable categórica, con la primera componente se encontró que las muestras de San Vicente de Chucurí (SAN VTE) presentaron ligeramente mayores valores de humedad, mientras que las muestras de Arauquita (ARAUQ) mostraron características de fermentación un poco mejores. Con respecto a la segunda componente, no hubo diferencias importantes entre localidades relacionadas con el índice de grano (IG) y la cantidad de granos bien fermentados (BF) (Fig. 7). 


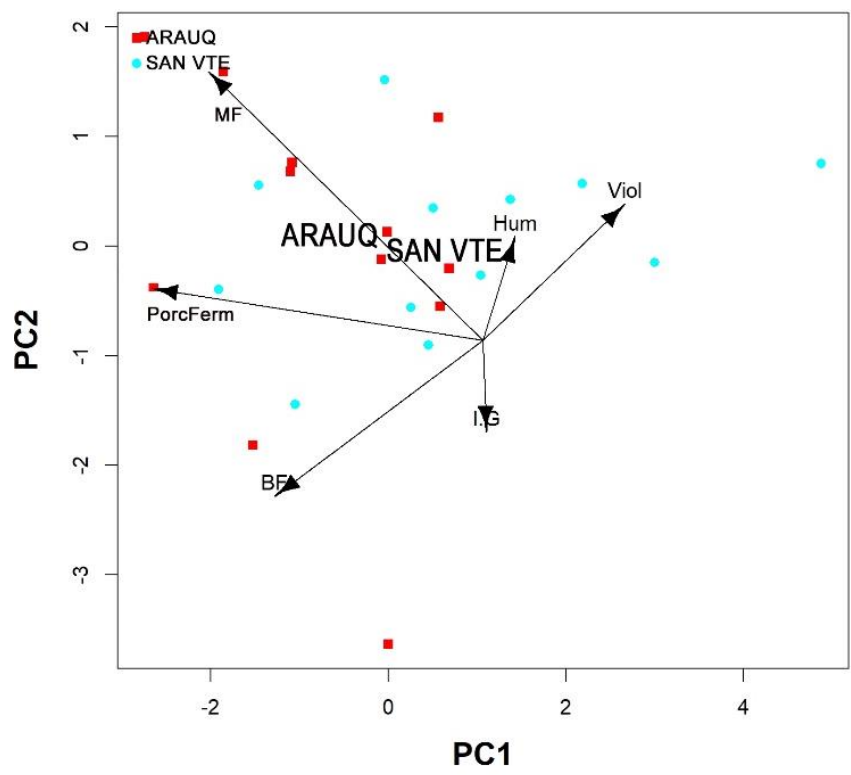

Fig. 7. Análisis de componentes principales - ACP-, componentes 1 y 2, para características físicas utilizando como variable categórica la localidad.

\section{CONCLUSIONES}

El análisis de componentes principales permitió evidenciar la correlación existente entre las características sensorialmente agradables (cacao, nuez, fruta fresca, fruta seca), así como entre las características poco apetecibles (amargor, acidez, astringencia).

Aun cuando las localidades evaluadas cuentan con condiciones agroecológicas diferentes, se demostró que los modelos tuvieron el mismo comportamiento. Las diferencias encontradas están ligadas al tipo de material utilizado.

Las características agradables obtenidas en los modelos se encuentran directamente relacionadas con el adecuado proceso cosecha y poscosecha, especialmente en la fermentación, que es la fase donde se forman los precursores del sabor y aroma de los granos de cacao, e igualmente están relacionadas con el genotipo utilizado.

El análisis sensorial de los cuatro modelos de siembra sirve de instrumento para la propuesta de ajustes a los protocolos en el proceso de fermentación y secado, especialmente para el material CCN 51, teniendo en cuenta las características presentadas por el M4 en las dos localidades, determinadas por sus bajos porcentajes de fermentación y altos contenidos de acidez, amargor y sensación de astringencia. 


\section{AgRAdECIMIENTOS}

Agradecimientos al panel de evaluación sensorial de la Federación Nacional de cacaoteros - FEDECACAO, Fondo Nacional del cacao, al grupo de investigación e innovación en cacao GIIC - FEDECACAO. Así mismo, a los técnicos de campo de las unidades técnicas Arauquita y San Vicente de Chucurí, por el apoyo en la realización de esta investigación.

\section{REFERENCIAS}

Afoakwa, E. O., Paterson, A., Fowler, M., \& Ryan. A. (2008). Flavor formation and character in cocoa and chocolate: a critical review. Critical Reviews in Food Science and Nutrition, 48(9), 840-857. https://doi.org/10.1080/10408390701719272.

Afoakwa, E. O., Kongor, J. E., Takrama, J. F., \& Budu, A. S. (2013). Changes in acidification, sugars and mineral composition of cocoa pulp during fermentation of pulp pre-conditioned cocoa (Theobroma cacao) beans. International Food Research Journal, 20(3), 1215-1222.

Aranzazu, F., Martínez, N., Palencia, G., Coronado, R., \& Rincón, D. (2009a). Cacao: manejo del recurso genético para incrementar la producción y productividad del sistema de cacao en Colombia. Bucaramanga, Colombia. Recuperado de http://hdl.handle.net/20.500.12324/19489.

Aranzazu, F., Martínez, N., Rincón, D., \& Palencia, G. (2009b). Materiales de cacao en Colombia, su compatibilidad sexual y modelos de siembra: unión temporal cacao de Colombia uno. Bucaramanga, Colombia. Recuperado de http://hdl.handle.net/20.500.12324/2353.

Boza, E. J., Motamayor, J. C., Amores, F. M., Cedeño, S., Tondo, C., Livingstone, D. S., Schnell, R., \& Gutiérrez, O.A. (2014). Genetic characterization of the cacao cultivar CCN 51: its impact and significance on global cacao improvement and production. Journal of the American Society for Horticultural Science, 139(2), 219-229. https://doi.org/10.21273/JASHS.139.2.219.

Brito, R. (2007). Características físico-químicas y organolépticas de cacao criollo en Venezuela. En Seminario Internacional de cacao, avances de investigación. Bucaramanga, Colombia.

Contreras, C., Ortiz, L., Graziani, L., \& Parra, P. (2004). Fermentadores para cacao usados por los productores de la localidad de Cumboto, Venezuela. Agronomía Tropical, 54(2), 219-232.

Crafack, M., Keul, H., Eskildsen, C. E., Petersen, M. A., Saerens, S., Blennow, A., Skovmand-Larsen, M., Swiegers, J. H., Petersen, G. B., Heimdal, H., \& Nielsen, D. S. (2014). Impact of starter cultures and fermentation techniques on the volatile aroma and sensory profile of chocolate. Food Res Int., 63, 306-316. https://doi.org/10.1016/j.foodres.2014.04.032.

De, Z. (2009). Cocoa \& Chocolate Manual. (40th Anniversary Edition). Switzerland: ADM Cocoa International.

Eskes, B., Ahnert, D., Assemat, S., García, L., Guarda, D., García, R. P., \& Seguine, E. (2012). Evidence on the effect of the cocoa pulp flavour environment during fermentation on the flavour profile of chocolates. En 17th International Cocoa Research Conference (COPAL), Yaoundé, Cameroun.

Enríquez, G. (1985). Curso sobre el cultivo del cacao. Turrialba, Costa Rica: Centro Agronómico Tropical de Investigación y enseñanza. CATIE.

Fisher, C., \& Scott. T. (2000). Flavores de los alimentos: biología y química. España: Acribia.

Guisande, C., Heine, J., González-DaCosta, J., \& García-Roselló, E. (2014). RWizard Software. Vigo, España: Universidad de Vigo. Recuperado de http://www.ipez.es/RWizard.

International Cocoa Organization -ICCO- (2016). Fine or Flavour Cocoa. Retrieved from https://www.icco.org/about-cocoa/fine-or-flavour-cocoa.html. 
Instituto Colombiano de Normalización y Certificación -ICONTEC- (2009). Norma Técnica Colombiana NTC 3929: Análisis Sensorial. Metodología. Métodos del perfil del sabor. Bogotá, D.C.: ICONTEC.

Instituto Colombiano de Normalización y Certificación -ICONTEC- (2012). Norma Técnica Colombiana NTC 1252: Cacao en grano. Bogotá, D.C.: ICONTEC.

Jiménez, J., Amores, F., Nicklin, C., Rodríguez, D., Zambrano, F., Bolaños, M., Reynel, V., Dueñas, A., \& Cedeño, P. (2011). Micro fermentación y análisis sensorial para la selección de árboles superiores de cacao. Boletín Técnico n. 140. Quevedo, Ecuador: INIAP, Estación Experimental Tropical Pichilingue. Recuperado de http://repositorio.iniap.gob.ec/handle/41000/1585

Martínez, N., Moreno, E., Duarte, D., \& Gavanzo, O. (2016). Nuevos clones regionales de cacao. Base de la biodiversidad, productividad y calidad del cacao de Colombia. Bucaramanga, Colombia: Federación Nacional de Cacaoteros.

Motamayor, J. C., Lachenaud, P., Da Silva, E., Mota, J. W., Loor, R., Kuhn, D. N., \& Brown, J. S. (2008). Geographic and Genetic Population Differentiation of the Amazonian Chocolate Tree (Theobroma cacao L). PLoS ONE, 3(10), e3311. https://doi.org/10.1371/journal.pone.0003311.

Quintana, L., Hernández, M., Rivera, M., \& Moreno, E. (2018). Evaluation of the Fermentation Time on the Physical Characteristics of Cocoa (Theobroma cacao L), Clones Fedecacao Tame 2, Fedecacao Lebrija 3 and Fedecacao Saravena 12 in the Town of San Vicente de Chucuri. Advance Journal of Food Science and Technology, 15(SPL), 184-190. https://doi.org/10.19026/ajfst.15.5892.

Sánchez, V. (2007). Caracterización organoléptica del cacao (Theobroma cacao L.), para la selección de árboles con perfiles de sabor de interés comercial. Ecuador: Universidad Técnica Estatal de Quevedo.

Stevenson, C., Corven, J., \& Villanueva, G. (1993). Manual para análisis de cacao en laboratorio. San José, Costa Rica: IICA.

Sim, S. Y., Ng, J. W., Ng, W. K., Forde, C. G., \& Henry, C. J. (2015). Plant polyphenols to enhance the nutritional and sensory properties of chocolates. Food Chemistry, 200, 46-54. https://doi.org./10.1016/j.foodchem.2015.12.092.

Tavares, A., Batista, N., Lacerda, C., Reis, A., Efraim, P., Marques, A., \& Schwan, R. (2016). Investigation of chocolate produced from four different Brazilian varieties of cocoa (Theobroma cacao L.) inoculated with Saccharomyces cerevisiae. Food Research International, 81, 83-90. https://doi.org/10.1016/j.foodres.2015.12.036. 